\title{
Le statut liminal du fœetus mort en France
}

Du « déchet anatomique » à l'« enfant »

The Liminal Status of Dead Fetuses in France

\section{Anne-Sophie Giraud}

\section{(2) OpenEdition}

Journals

Édition électronique

URL : https://journals.openedition.org/tc/7790

DOI : $10.4000 /$ tc. 7790

ISSN : 1952-420X

Éditeur

Éditions de l'EHESS

\section{Édition imprimée}

Date de publication : 31 octobre 2016

Pagination : 60-63

ISBN : 9782713225291

ISSN : 0248-6016

\section{Référence électronique}

Anne-Sophie Giraud, « Le statut liminal du fœtus mort en France », Techniques \& Culture [En ligne], 65-66 | 2016, mis en ligne le 06 janvier 2017, consulté le 29 septembre 2022. URL : http://

journals.openedition.org/tc/7790 ; DOI : https://doi.org/10.4000/tc.7790 


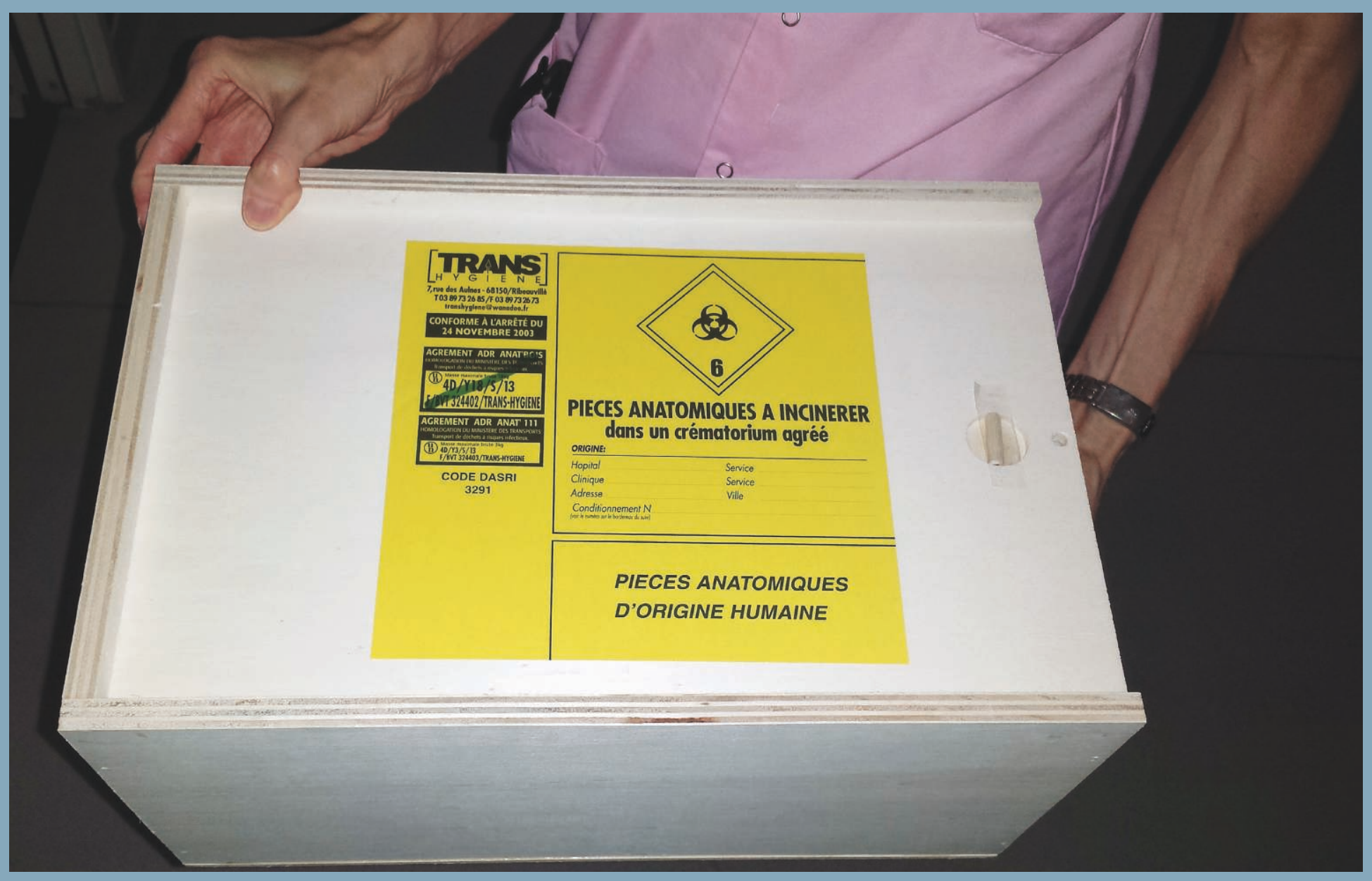




\section{Le statut liminal du fœtus mort en France} Du « déchet anatomique » à l'« enfant "

Depuis les années 1990, en France comme dans l'ensemble des pays Européens ${ }^{1}$, le statut juridique du fotus mort et les lois autour du traitement de son corps se sont profondément transformés. Ils n'ont eu de cesse de se consolider dans le sens d'une «personnalisation». Le scandale provoqué par la découverte des corps de fotus à l'hôpital Saint-Vincent-de-Paul en 2005 a révélé l'ampleur de cette mutation. Alors qu'il était considéré comme un « déchet» voire une "pièce de collection anatomique» depuis le xixe siècle sans provoquer de réactions particulières (Morgan 2009), le fotus mort est désormais perçu comme un être devant faire l'objet d'une considération particulière, tant de la part des professionnels que des «parents».

Ces transformations ont en particulier affecté le foetus des «états intermédiaires », à savoir celui né vivant ou mort entre 14 et 22 semaines d'aménorrhée (SA) ${ }^{2}$, ainsi que le foetus mort in utero au-delà de 22 SA. Avant 1993, le corps de ce foetus était considéré comme un « déchet anatomique ${ }^{3}$ », au même titre que des fragments d'organes auxquels il était assimilé. Le devenir du corps du fotus dépend essentiellement de l'établissement d'un certificat médical d'accouchement et d'un acte d'«enfant sans vie». Ces documents ouvrent la possibilité aux couples, s'ils le désirent, de réaliser des obsèques. Or, ce statut juridique d'« enfant sans vie» n'a cessé de s'élargir à des pertes de plus en plus précoces: le seuil limite de déclaration fixé d'abord à 28 SA en 1993, abaissé ensuite à 22 SA en 2001, est enfin supprimé en 20084 . Ainsi, les seuils qui inscrivent le foetus dans l'une ou l'autre de ces catégories, celle de « déchet anatomique », de «pièce anatomique» et de "personne décédée» mais aussi, plus importante, d'«enfant sans vie», ont été progressivement redéfinis. Nous assistons à un élargissement du statut de l'«enfant sans vie», alors que celui du « déchet» ne cesse de se réduire.

Avant les années 1990, il était également impensable, et ce quel que soit le terme, de le montrer aux couples. Ces pratiques hospitalières ont été complètement bouleversées. Le corps du fœetus mort fait désormais l'objet d'une attention particulière. Il est lavé, vêtu et présenté 
aux couples comme un enfant. C'est même cette humanisation progressive de ses « restes » qui a contraint le droit à certains arrangements (Memmi 2011).

La particularité d'une telle mort, par ailleurs autrefois considérée comme un phénomène «infrasocial» (Hertz 1906), est qu'elle ne laisse que très peu de «restes» matériels et mémoriels. Or, suite à ces changements, certains couples endeuillés se lancent dans une entreprise intense de création de souvenirs, de stratégies et de bricolages divers, pour faire exister cet être et continuer d'entretenir une relation étroite avec lui (autels domestiques, photographies, etc.). Le don au défunt occupe une place centrale. Il permet de s'instituer comme «parent» de cet «enfant», là où le plus grand nombre voit seulement un «accident de reproduction».

Cependant l'étude - en parallèle du statut juridique du fotus mort - des pratiques des professionnels (professionnels du soin et du funéraire) ainsi que des pratiques des couples endeuillés révèle que ces transformations - qui donnent l'impression d'une institution de la personne du foetus - sont en réalité bien plus complexes. Même s'il est désormais présenté à ses «parents » comme un «enfant», ses funérailles, lorsqu'elles ne sont pas prises en charge par la famille, ne sont toujours pas celles d'un nouveau-né et son corps subit invariablement le traitement octroyé aux « pièces anatomiques». On découvre finalement que le foetus mort ne bénéficie que d'un statut juridique et social liminal dont témoigne le traitement général de son corps et de ses «restes». Cela laisse une large place au choix et en particulier à la « volonté parentale», car le statut de son corps oscille sans cesse de «pièce anatomique» à «enfant», selon le sens que donnent les acteurs à leur relation avec cet être.

À travers l'étude des pratiques institutionnelles et privées, nous montrons que depuis les années 1980, la société française tend à voir émerger un statut nouveau accordé au fotus mort: il ne peut être considéré ni comme une personne ni comme une chose, mais comme un être spécifique. Ce statut se constate au niveau juridique mais aussi dans les pratiques sociales. Il se met en effet en place de façon complexe, non sans tensions, et il n'est pas sans rappeler certains statuts intermédiaires étudiés par des anthropologues tels qu'Arnold Van Gennep (1981) ou encore Agnès Fine (1994) en Europe. Il a pour particularité que, dans nos sociétés modernes «individualistes», ce statut s'organise autour d'une diversité offrant une possibilité de choix pour les individus - traduit par la «volonté parentale»-mais encadrée de manière stricte par le droit.

\section{En ligne}

Retrouvez l'article complet sur revues.org, TechniquesECulture 65-66 « Réparer le monde. Excès, reste et innovation »: http://tc.revues.org.

\section{Notes}

1. «Les enfants nés sans vie», les documents de travail du Sénat, série Législation Comparée, avril 2008.

2. 14 SA : limite légale de l'avortement. 22 SA: seuil de viabilité.

3. Il faut différencier le « déchet anatomique», non aisément identifiable par un non spécialiste (ex: fragments d'organes, fotus en deçà de 14 SA), et la «pièce anatomique», aisément identifiable par un non 
spécialiste (ex: membres amputés, fœetus au-delà de 14 SA). Elles ne bénéficient pas du même traitement.

4. Sous condition de la délivrance d'un certificat médical d'accouchement, nécessaire à l'établissement d'un acte d'enfant sans vie, impliquant le recueil d'un corps formé et sexué. Les situations en deçà de 14 SA ne répondent pas à ces conditions.

\section{I'auteure}

Anne-Sophie Giraud est anthropologue. Elle a soutenu sa thèse à l'École des hautes études en sciences sociales au Centre Norbert Elias (UMR 8562). Son travail porte sur le statut relationnel de l'embryon et du fotus en France. Depuis 2016, elle est post-doctorante au CeRIES (EA 3589) à l'Université de Lille 3 sur deux projets concernant les maladies pulmonaires rares, les maladies génétiques, la maladie chronique, le handicap et la transplantation. Elle travaille sur la parenté, l'assistance médicale à la procréation (AMP), l'anthropologie de la santé, les études féministes et la mort.

\section{Iconographie}

Image d'ouverture. Boîte destinée à recevoir des « pièces anatomiques», aussi bien des fœtus que des membres amputés. Elle porte le sigle DASRI (Déchets d'Activités de Soin à Risque Infectieux). (C) Anne-Sophie Giraud.

1. (C) Anne-Sophie Giraud.

\section{Références}

Fine, A. 1994 Parrains, marraines. La parenté spirituelle en Europe. Paris: Fayard.

Hertz, R. 1906 « Contribution à une étude des représentations collectives de la mort », L’Année sociologique $10: 48-137$.

Memmi, D. 2011 La Seconde vie des bébés morts. Paris Éditions de l'EHESS.

Morgan, L.M. 2009 Icons of Life. A Cultural History of Human Embryos. Berkeley: University of California Press.

Van Gennep, A. 1981 Les Rites de passage. Étude systématique des rites. Paris: A. et J. Picard.

\section{Pour citer cet article}

Giraud, A.-S. 2016 «Le statut liminal du foetus mort en France. Du "déchet anatomique" à l'“enfant" », Techniques ECulture 65-66 «Réparer le monde. Excès, reste et innovation», p. 60-63.

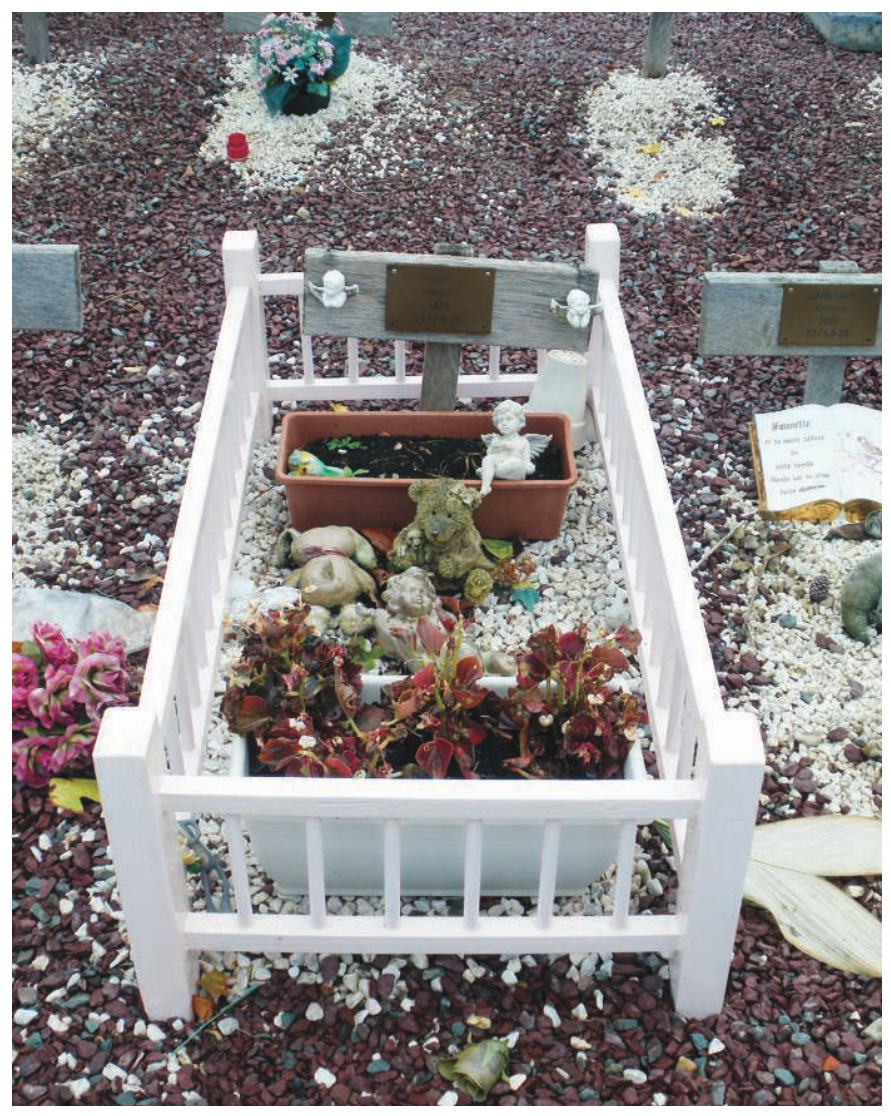

1. Tombe d'un enfant mort-né, ceinte de barreaux de berceau et décorée de nombreux jouets pour enfants. 